\title{
Concepts for estimating the fatigue strength of sintered steel components
}

\author{
Sebastian Götz a and Klaus-Georg Eulitz \\ TU Dresden, Faculty of Mechanical Science and Engineering, Institute of Solid Mechanics (IFKM), \\ 01062 Dresden, Germany
}

\begin{abstract}
The fatigue notch effect can be estimated using fracture mechanics-based support factors. Stress intensity factors for cracks in notches must therefore be calculated. There is a problem of transferability when $2 \mathrm{D}$ reference geometries are used for this. This can be avoided when modelling 3D cracks in the notch of the actual part to be assessed. This is more laborious, but leads to better results, which will be shown in this presentation. The questions of aspect ratio and to point on the crack front, where stresses are evaluated, will be discussed. The approach is validated using a broad database of different sintered steels. The statistical evaluation shows an almost exact prediction of the mean value with a relatively small scatter.
\end{abstract}

\section{Introduction}

The fatigue notch effect describes the difference in the fatigue strength of unnotched material specimens and notched parts. Support factors can be used to transfer the fatigue strength of the material to the actual part. There are different approaches for this. In a previous study [1] different concepts to estimate the fatigue strength of notched parts were examined and statistically evaluated based on a broad database of various steel-based powder metallurgical materials. As a result, the Theory of Critical Distances (TCD) led to a good prediction result, while the fracture mechanics-based approach led to a higher scatter and an overestimation of the fatigue strength. This presentation aims to explain the reasons for this difference and show how the weaknesses of the 2D facture mechanics-based approach can be overcome and thus lead to similarly good results as the TCD.

\section{Fracture mechanics-based approaches}

The small crack correction $a_{0}$ according to El Haddad [2] is used as reference crack size. It is calculated using the material's endurance limit and the threshold value for fatigue crack growth. Its magnitude is a measure for the material's sensitivity to notches. A common feature of the concepts is the calculation of the stress intensity factor at a crack of the length $\mathrm{a}_{0}$ in a reference notch (e.g. [3, 4]). The stress intensity

\footnotetext{
${ }^{a}$ Corresponding author: goetz_sebastian@yahoo.com
}

This is an Open Access article distributed under the terms of the Creative Commons Attribution License 4.0, which permits unrestricted use, distribution, and reproduction in any medium, provided the original work is properly cited. 

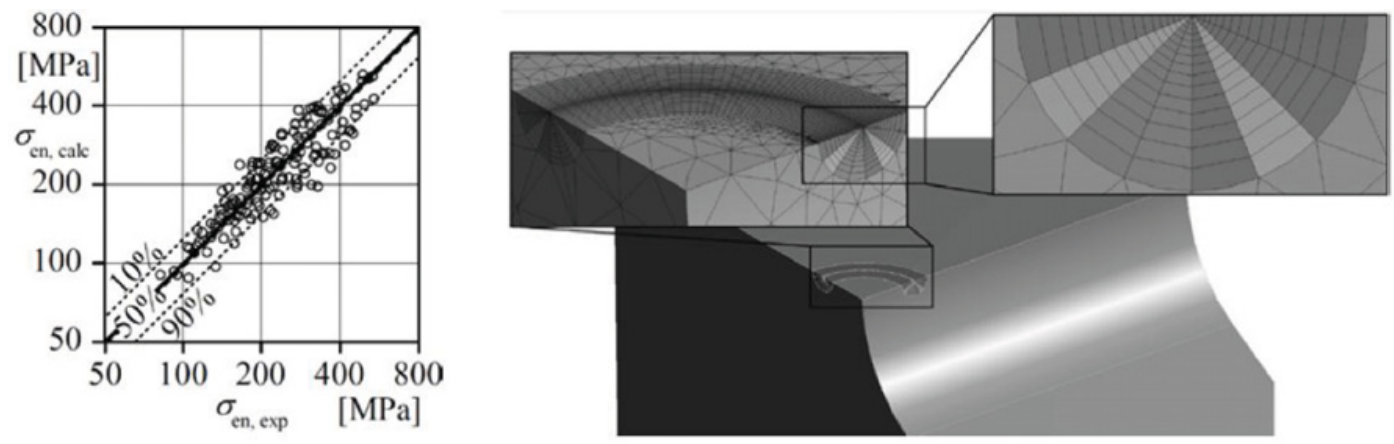

Figure 1. Comparison with experimental results (left), modelling of the crack in the notch root (right).

factor or the corresponding geometry function is calculated based on a known solution of a $2 \mathrm{D}$ reference notch. To apply the concepts to a specific component, its notch geometry and thus the surrounding stress field must be transferred to the reference notch. The reference notch must be represented adequately to assess the actual notch geometry. Characteristics such as the notch radius, the stress concentrations factor or the notch depth are used. This results in a problem of transferability, which involves a source of error and uncertainty.

\section{Support factor using 3D crack modelling}

If a support factor is to be applicable for arbitrarily shaped parts it cannot depend on the transferability to 2D reference geometries. In this study cracks are modelled in the notch using Finite Element analysis. An effective stress intensity factor normalized with the maximum principle stress in the notch is used as stress parameter:

$$
\psi_{I}\left(a_{0}, s\right)=K_{I}\left(a_{0}, s\right) / \sigma_{\mathrm{I}, \max } .
$$

Its value varies along the crack front and only depends on the material for a given notch and loading. The support factor $n$, which is the ratio of the endurable stress of a notched part $\sigma_{e, n}$ and of an unnotched specimen $\sigma_{e, 0}$ is calculated as follows:

$$
n=\sigma_{\mathrm{e}, \mathrm{n}} / \sigma_{\mathrm{e}, 0}=\psi_{\mathrm{I}, \max , 0}\left(a_{0}\right) / \psi_{\mathrm{I}, \max , \mathrm{n}}\left(a_{0}\right) .
$$

The value $\psi_{\mathrm{I}, \mathrm{max}}$ is the maximum that appears near the intersection of the crack front with the free surface for a crack in the notch root. The shape of the modeled cracks $a_{0}$ is semi-circular. The reason for this will be explained in more detail in the presentation. The parameter $a_{0}$ for the material in question has to be determined iteratively, by modelling cracks in the unnotched specimen used to determine the material's endurance limit using the condition:

$$
\psi_{\mathrm{I}, \max , 0}\left(a_{0}\right)=\Delta K_{\mathrm{I}, \mathrm{th}} /\left(2 \cdot \sigma_{\mathrm{e}, 0}\right) .
$$

\section{Validation with experimental results}

The database from [1] was used for validation. It comprises 153 experimentally obtained support factors, from 17 different $\mathrm{P} / \mathrm{M}$ steels varying in chemical composition and density. In contrast to the $2 \mathrm{D}$ approach, the mean value of the prediction is nearly exact and the scatter is smaller. 
FDMDII - JIP 2014

\section{References}

[1] S. Götz, K.-G. Eulitz, Int J Fatigue 52, 1-10 (2013)

[2] M. H. El Haddad, K. H. Smith, T. H. Topper, J Eng Mater Technol 101(1), 42-46 (1979)

[3] W. Lukáš et al., Fatigue Fract Eng Mater Struct 9(3), 195-204 (1986)

[4] Y. Liu, S. Mahadevan, Eng Fract Mech 76, 2317-2331 (2009) 